\title{
The feasibility of a primary care model for the management of COPD
}

\author{
Marianne A. Meulepas a,*, Johanna E. Jacobs ${ }^{a}$, Annelies E.M. Lucas ${ }^{b}$, \\ Frank W.J.M. Smeenk ${ }^{c}$, Ivo Smeele ${ }^{d}$, Ben J.A.M. Bottema ${ }^{a}$, \\ Richard P.T.M. Grol ${ }^{a}$
}
a Centre for Quality of Care Research (WOK), University Medical Centre, PO Box 9101, 6500 HB Nijmegen, The Netherlands
b Department of Family Medicine, Maastricht University, The Netherlands
c Department of Pulmonology, Catharina Hospital, Eindhoven, The Netherlands
d COPD \& Asthma Primary Care Group (CAHAG), Utrecht, The Netherlands

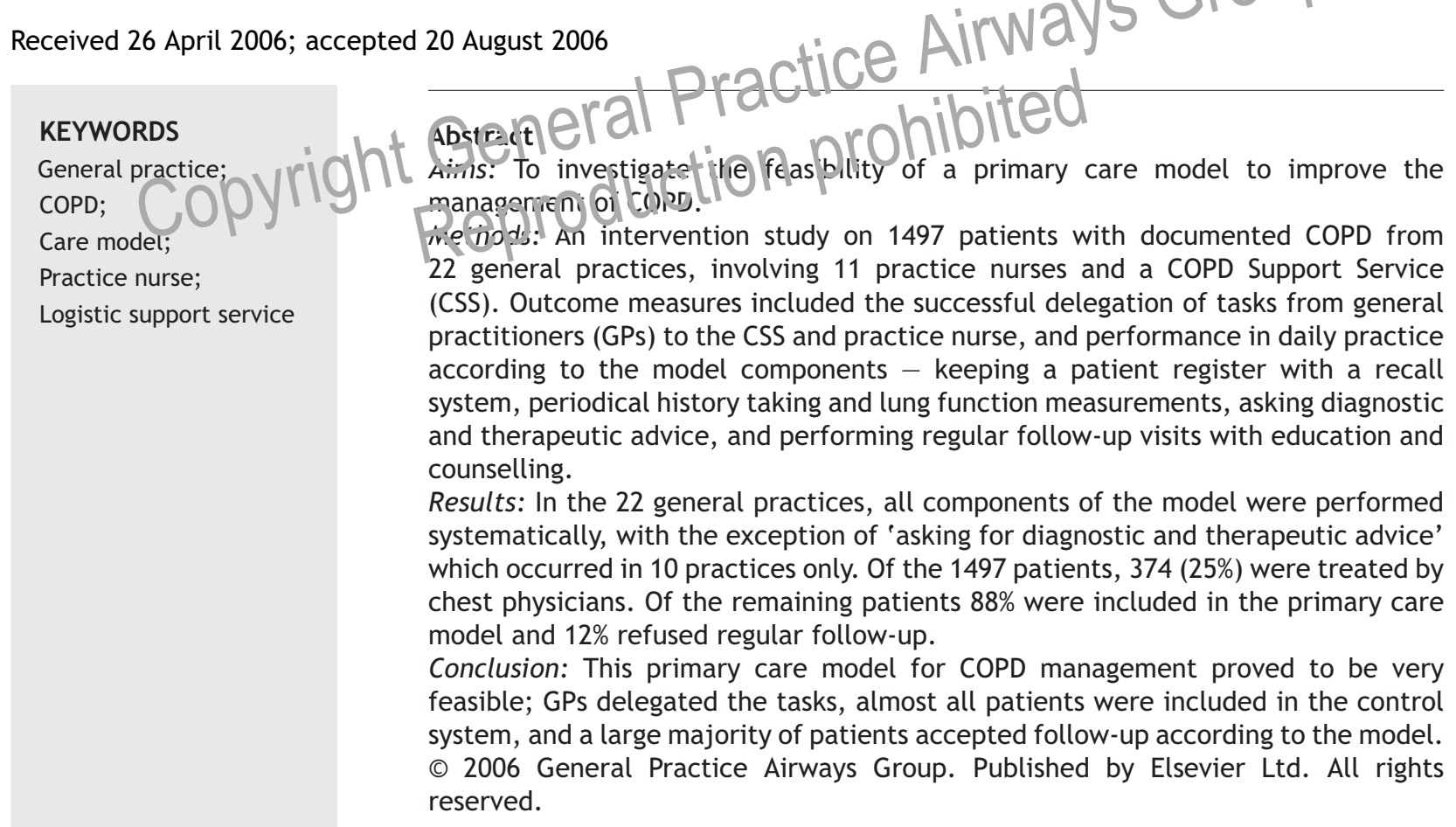

* Corresponding author. Tel.: +3124 3616338; fax: +31 243540166 .

E-mail address: m.meulepas@kwazo.umcn.nl (M.A. Meulepas).

\section{Introduction}

Chronic obstructive pulmonary disease (COPD) is a chronic disease with a broad spectrum of 
severity and a large number of patients who have less severe disease. Care for this group of patients can best be provided in primary care, at least in terms of continuity, comprehensiveness and coordination [1]. To that end, internationally accepted guidelines have been developed and distributed [2,3]. It is recommended that clinicians use lung function measurements when diagnosing and monitoring COPD, checking health status and symptoms, and if necessary, adjusting medication. Furthermore, education should be used to help patients take over the daily management of their disease. Periodical contact appears to have a positive effect on long-term patient outcomes and quality of life [4].

In the Netherlands, the usual management of COPD patients is not optimal; in particular, regular follow-up visits and periodical lung function measurement are often not offered $[5,6]$. For general practice care to meet guideline recommendations, a number of factors are required which are often lacking [7-12]. These are: (1) decision support; (2) registries and reminder systems to ensure active follow-up; and (3) patient education and self-management support. Decision support means introducing specialist expertise for consultation in diagnosis and treatment [13]. A register is a list of all patients with a speclie chronic disorder from which the carerleeded for each patient can be $p$ donned an active recall system spots roing at er clees, so steps car bel taken to contaci tnuse patients 12. seli-mariagement education is provided to increase self-efficacy for improving clinical outcomes [15]. These three

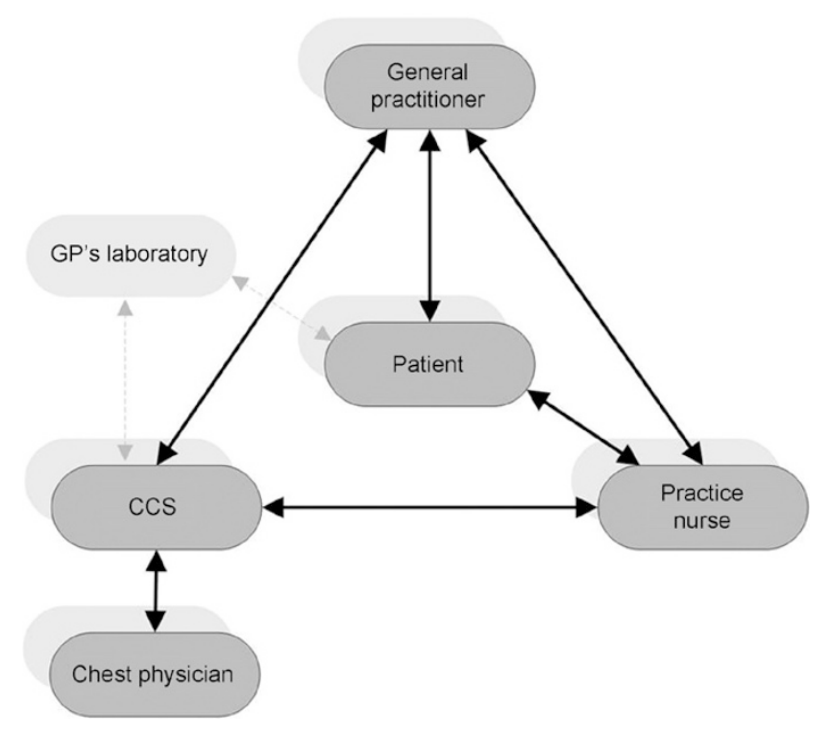

Figure 1 Actors primary care model for COPD. factors all require a different type of expertise. Decision support has to be given by a medical expert, while registries for follow-up and patient self-management education include major roles for non-physicians [16-19].

We have developed a primary care model that integrates these different factors which require specific expertise (Figure 1). The implications of this model are twofold. On the one hand, it aims to help general practitioners (GPs) provide care according to guidelines. On the other hand, it needs to be accessible and attractive to patients so that they will participate. In this study the feasibility of the model in daily practice was tested.

\section{Methods}

\section{Study population}

This study evaluates the two-year implementation of a primary care disease management model in 22 practices (involving 29 GPs) in the South of the Netherlands. In the one-year run-in period, practice nurses were trained and the COPD Support Service (CSS) was organised. All 149 practicel: in the region were invited to partictnate by oper invitation. General pect cos cualified for the care model if ct $\in$ Junet various inclusion criteria: co-operation with other mact 65 to reach a total population of at lesst 4500 patients; a working space for the prictice nurse; and the use of an electronic medical register. Of the 81 practices that showed interest, 44 met the inclusion criteria. Based on regional distribution criteria 22 practices were allocated to the intervention group.

After 18 months, the 22 practices had appointed 11 practice nurses with 2.1 nurses per 1000 COPD patients.

All patients with documented COPD after two years intervention (documentation is part of the intervention) in these 22 practices were included in the study.

\section{Model-see Box 1}

\section{Measures and data collection}

Data collection took place 18 months after the general practices started to implement their own protocol. The following data were collected to answer the research question concerning implementation of the model: the percentage of practices (1) with a patient register and recall system; (2) performing periodical case history reporting and lung function measurements; (3) asking for diagnostic and therapeutic advice; (4) arranging periodical visits; and (5) providing 


\section{Box 1: Primary Care Model}

The GP can delegate tasks to the practice nurse and the CSS but remains responsible in the end. To that purpose, every general practice will make a practice protocol in which the division of roles is described.

The CSS is a logistic support service, connected to the regional primary care laboratory with a specialised lung nurse and some administrative employees. It offers logistic support to the general practice by means of managing the patient register and a recall system for annual history taking and lung function measurement (performed by the primary care laboratory if not performed in the practice itself). The CSS also forms the link with the chest physician.

A number of chest physicians from the regional hospitals provide diagnostic and therapeutic advice by evaluating the lung function measurements and case history reports (without actually seeing the patient).

The GP discusses the results of the check-up with the patients and determines whether the medication is still adequate.

The practice nurse's most important tasks are education and counselling. Patients who meet the referral criteria will be referred to a chest physician for fur the cia sim sis or treatment.

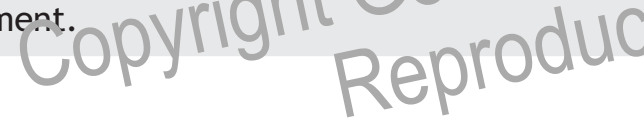

advice and counselling according to the protocol. The following data were collected to determine the degree of inclusion in the care model: the percentage of COPD patients treated by the GP who were included in the care model.

Data were collected through documents (protocols), interviews, the CSS and general practice registration (inclusion patients). The following targets were set as criteria for success:

- in all the participating practices $(100 \%)$ the five elements of the care model are part of their own protocol;

- at least $65 \%$ of the patients treated by the GP are included in the model; this level was chosen because a drop-out of 40 to $60 \%$ is described in the literature in patients attending comparable programs for asthma care $[20,21]$.

\section{Analysis}

The data about the usage of the model and the inclusion of patients were processed in frequencies, percentages and averages.
Table 1 Characteristics of practices at baseline (sd)

\begin{tabular}{ll}
\hline Number of practices & 22 \\
Number of general practitioners & 29 \\
$\%$ urban practice $(>80.000$ inhabitants) & 38 \\
$\%$ single handed practices & 27 \\
Mean population/FTE & $2519(346)$ \\
$\%$ practices with active recall system & 0 \\
\hline
\end{tabular}

\section{Results}

\section{Population}

The study was performed amongst 22 practices with a total number of $29 \mathrm{GPs}$, of whom $76 \%$ were male (Table 1). At the start of the study, the average age of the GPs was 46 years; $38 \%$ of the study practices were located in an urban area $(>80,000$ inhabitants) and $32 \%$ were single-handed. None of the practices had a patient follow-up register at baseline.

\section{Implementation of the model}

In all participating practices all components of the model were performed susterimaticaliy, with the exception of /aikingl fo: i iaynostic and therapeutic adv ce which occurred in only 10 practices - see ráble 2.

Afte t t $\in$ study a double patient register existed for $\in$ ach practice. The practice nurse kept a list of all the patients with documented COPD and the CSS had their own register of patients who had to be called for a check-up. In seven practices the laboratory performed the periodical history taking and lung function measurement, and 15 practices performed these tests on their own.

In all the practices the practice nurse set up a COPD clinic. In those sessions she integrated discussing test results and medication counselling with education and counselling. She discussed the test results with the GPs before discussing them with the patient. The patient was only seen by the GP if there were special circumstances or symptoms which required assessment.

\section{Degree of inclusion in the care model}

Altogether, the 22 general practices saw a total of 1497 patients with documented COPD in the first 18 months of the study (prevalence 2.4\%). Of these patients 374 were referred to the chest physician, leaving 1123 patients under the control of the general practices (Figure 2).

Prior to the intervention none of the practices had an active monitoring system. Eighteen months later $81 \%$ of the 1123 patients were included 
Table 2 Model components implemented

\begin{tabular}{|c|c|c|c|c|}
\hline \multirow[t]{2}{*}{ Model components } & \multicolumn{4}{|c|}{ Care taker (in n practices): } \\
\hline & PN & $\mathrm{CSS} / \mathrm{CP}$ & LAB & Total \\
\hline Patient register with all the diagnosed patients & 22 & & & 22 \\
\hline Patient register for recall system & 13 & 9 & & 22 \\
\hline Periodical anamnesis and spirometric test & 15 & & 7 & 22 \\
\hline Diagnostic and therapeutic advice & & 10 & & 10 \\
\hline Periodical check-up & 22 & & & 22 \\
\hline Self-management education & 22 & & & 22 \\
\hline
\end{tabular}

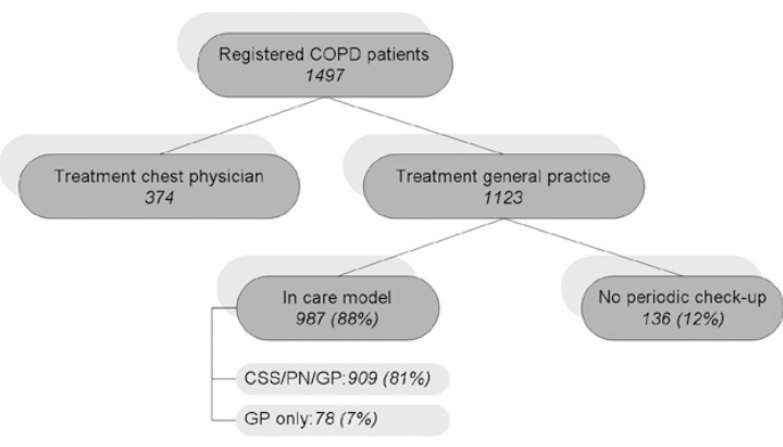

Figure 2 Division of patients in and outside care model.

in the care model with periociolung function measurement followed by a visic to the practice nurse. (of the patients seen by the GPo (1) 40 did not need perrodic monitoring; 2: had co-morbidity, and in 15 patients the diagnosis had not been confirmed.

Altogether, 136 patients eligible for admission to the care model refused periodic monitoring.

\section{Discussion}

This primary care model for COPD appears to be very applicable to other general practice settings. The GP delegated tasks to the CSS and the practice nurse according to the model, and almost all patients were included in the control system. A large majority of patients accepted the frequent check-ups as per the model. The prevalence of COPD in the study group was 23.6 per 1000 which corresponds to the annual prevalence among the Dutch population (20.7 per 1000) calculated from nine different GPs' registries [22]. The distribution of the care between GP and medical specialist, 75\% versus $25 \%$, is difficult to compare with the Dutch situation. In 2003, 78\% of COPD patients visited the chest physician, but we do not know if they were treated in shared care [23].
The findings are difficult to compare with other study results because we could not find a similar care model in the literature. Studies on the use of a practice nurse are nearly always focussed on the effects on patient outcome parameters such as lung function, use of medication, symptoms, and quality of life, and not on participation in planned care. However, we can compare our study with a programme which was aimed at implementing guidelines into general practice in vich $23 \%$ of the patients dropped o(II witr in one year [24]. In compariso i the baticipation of patients in our Calle niedel is considerably higher; only $136(12 \%)$ of the eligiblectict $t$ were not followed-up within the dae modiel because they refused to take part. With the introduction of the model in participating practices, patient registration was, for the first time, set up on the basis of clear (repeated) diagnostic parameters. This in itself should be seen as an enormous improvement in quality. The model provides insight into the prevalence figures for each practice and forms the basis for further monitoring of COPD patients. For the first time patients are being monitored and supported systematically.

Various components of the model - such as having a patient register with a recall system; periodical history taking and lung function measurement; asking diagnostic and therapeutic advice; and periodical patient visits with information and counselling - appear to fit very well into one model. The model equips general practices with facilities to meet COPD guidelines. Barriers to organising planned visits and periodic lung function measurement disappeared. This appears to be a promising model that offers general practices the opportunity to follow guidelines in a modern context [25]. Further research is needed to study the influence of this primary care-based disease management model on the health status of COPD patients. 


\section{Conflict of interest}

There was no conflict of interest for the authors in the preparation of this article.

\section{Acknowledgement}

Funding. This study was funded by the Dutch Asthma Foundation and the Health Insurance companies CZ and VGZ.

\section{References}

[1] Starfield B, Lemke KW, Bernhardt T, Foldes SS, Forrest CB, Weiner JP. Comorbidity: implications for the importance of primary care in 'case' management. Ann Fam Med 2003;1(1):8-14.

[2] GOLD guidelines for the management of COPD (www.goldcopd.com).

[3] Buist AS. Guidelines for the management of chronic obstructive pulmonary disease. Respir Med 2002;96(Suppl C):S11-6. Review.

[4] Nishiyama $\mathrm{O}$, Taniguchi $\mathrm{H}$, Kondoh $\mathrm{Y}$, et al. Factors in maintaining long-term improvements in health-related quality of life after pulmonary rehabilitation for COPD. Qual Life Res 2005;14(10):2315-21.

[5] Jans MP, Schellevis FG, Le Coq EM, Bezemer PD, van Eijk JT. Health outcomes of asthma and COPD patien is: the evaluation of a project to implement suder nes in general practice. Int J Qual realt $2(a)$ i $201 ; 13(1)$ : $17-25$.

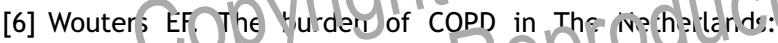
results crom the Confronting ( $D$ [ o r 0 ey. Respir Med 2003;97(Suppl C):S51-9.

[7] Von Korff M, Katon W, Unutzer J, Wells K, Wagner EH. Improving depression care: barriers, solutions, and research needs. J Fam Pract 2001;50(6):E1. Review.

[8] Javors JR, Bramble JE. Uncontrolled chronic disease: patient non-compliance or clinical mismanagement? Dis Manag 2003;6(3):169-78.

[9] Worth H, Dhein Y. Does patient education modify behaviour in the management of COPD? Patient Educ Couns 2004;52(3):267-70. Review.

[10] O'Dowd LC, Fife D, Tenhave T, Panettieri Jr RA. Attitudes of physicians toward objective measures of airway function in asthma. Am J Med 2003;114(5): $391-6$.
[11] Palen van der J, Monninkhof E, Valk van der P, Visser A. Managing COPD: no more nihilism! Patient Educ Couns 2004;52(3):221-3.

[12] Barnes PJ. Asthma guidelines: recommendations versus reality. Respir Med 2004;98(Suppl A):S1-7.

[13] Bodenheimer T, Wagner EH, Grumbach K. Improving primary care for patients with chronic illness. JAMA 2002;288(14):1775-9.

[14] Rundall TG, Shortell SM, Wang MC, Casalino L, Bodenheimer T, Gillies RR, et al. As good as it gets? Chronic care management in nine leading US physician organisations. BMJ 2002;325(7370):958-61.

[15] Bodenheimer T, Lorig K, Holman H, Grumbach K. Patient self-management of chronic disease in primary care. JAMA 2002;288(19):2469-75.

[16] Rothman AA, Wagner EH. Chronic illness management: what is the role of primary care? Ann Intern Med 2003;138(3):256-61. Review.

[17] Veale BM. Meeting the challenge of chronic illness in general practice. Med J Aust 2003;179(5):247-9.

[18] Lindberg M, Ahlner J, Moller M, Ekstrom T. Asthma nurse practice-a resource-effective approach in asthma management. Respir Med 1999;93(8):584-8.

[19] Musto PK. General principals of management: education. Nurs Clin North Am 2003;38(4):621-33. Review.

[20] Muntner P, Sudre P, Uldry C, Rochat T, Courteheuse C, Naef $\mathrm{AF}$, et al. Predictors of participation and attendance in a new asthma patient self-management education program. Chest 2001;120(3):778-84.

[21] Abdulwadud O, Abramson M, Fordes A Jaines A, Light $L$, Thien $F$, et al. Attondare at =n aschma educational intervention (chalce is ics of participants and nonba ticipants. Respir Med 1997;91(9):524-9.

122y Poos MJJG, Gi.sf.n. Prevalentie, incidentie en

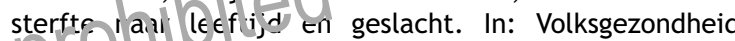
lof lo.nst Verkenning, Nationaal Kompas Volksgezondheid. Bilthoven: RIVM, <http://www.nationaalkompas.nl> Gezondheid en ziekte $\backslash$ Ziekten en aandoeningen $\backslash$ Ademhalingswegen $\backslash$ Astma en COPD, 22 november 2004.

[23] Heijmans MJWM, Spreeuwenberg P, Rijken PM. Monitor Zorg- en Leefsituatie van mensen met astma en mensen met COPD - Trends en ontwikkelingen over de periode 2001-2004. NIVEL - Nederlands instituut voor onderzoek van de gezondheidszorg, mei 2005.

[24] Jans MP, Schellevis FG, Le Coq EM, Bezemer PD, van Eijk JT. Health outcomes of asthma and COPD patients: the evaluation of a project to implement guidelines in general practice. Int J Qual Health Care 2001;13(1):1725.

[25] Showstack J, Lurie N, Larson EB, Rothman AA, Hassmiller S. Primary care: the next renaissance. Ann Intern Med 2003;138(3):268-72.

Available online at www.sciencedirect.com

ScienceDirect 